RU-95-29

\title{
The Dual of Supersymmetric SU(2k) with an Antisymmetric Tensor and Composite Dualities
}

\author{
M. Berkooz * \\ Department of Physics and Astronomy \\ Rutgers University \\ Piscataway, NJ 08855-0849
}

\begin{abstract}
We suggest a dual to an $S U(2 k)$ Susy gauge theory containing an antisymmetric tensor, $n_{F}$ fundamentals and $n_{\bar{F}}$ anti-fundamentals. This is done by expanding the theory into an equivalent description with two gauge groups and then performing known duality tranformations on each gauge group separately. Chiral operators, mass perturbations and flat directions are discussed.
\end{abstract}

$5 / 95$

* e-mail: berkooz@physics.rutgers.edu 


\section{Introduction}

As has been recently demonstrated, the holomorphic structure of Susy gauge theories allows one to calculate some of their I.R. properties exactly [1] [3 [16] (for earlier work see [2] [18 20]). In particular some of them can be given an equivalent low energy description that interchanges weak and strong coupling regions, generalizing the Olive-Montonen electric-magnetic duality [21]. Most existing $N=1$ examples of this duality have a common flavor to them in the sense that the dual model is similar to the original model with a different number of colors and additional gauge invariant fields. There is, however, no necessity that this will be the general case. Accepting the notion that non-abelian gauge degrees of freedom are almost fictitious in the infra-red, one may have a dual, or equivalent description, with an arbitrary gauge groups structure.

We will suggest that an $S U(2 k)$ Susy gauge theory with an antisymmetric tensor and appropriate number of quarks and anti-quarks has an equivalent description in terms of a theory with two gauge groups and therefore a chain of duals with two gauge groups. By construction, anomaly matching is immediate and so is partial identification of the chiral ring. We also discuss mass perturbations and flat directions.

\section{The Electric Theory}

The theory in question is an $S U(N=2 k)$ gauge theory with $n_{F}$ quarks $(Q)$ in the fundamental rep., $n_{\bar{F}}$ antiquarks $(\bar{Q})$ in the anti-fundamental rep., and an antisymmetric tensor $(A)$ [2]. Anomaly cancelation requires $N=n_{\bar{F}}-n_{F}+4$.

There are two ordinary $\mathrm{U}(1)$ global symmetries and one $U(1)_{R}$. We will be interested, however, in the theory with a superpotential $W=\operatorname{Pf}(A)$. Let us assume that we do not add this superpotential and discuss the status of the operators $\operatorname{Pf}(A) Q A^{-1} Q$. By the following pragmatic and very porous argument, we have to set these operators to zero.

There are 3 options:

1. $\operatorname{Pf}(A) Q A^{-1} Q$ is elementary in the dual theory. Let us look at the limit $n_{F}, n_{\bar{F}} \rightarrow$ $\infty$ at a fixed ratio. Some $\sum U(1)$ anomaly term will scale like $N^{3}$ and $\sum U(1)^{3}$ will scale like $N^{5}$. It is unclear how to cancel these contributions. We are justified in taking this 
limit since the equations for the anomaly matching are algebraic. If the anomalies match in a limited region of $n_{F}$ and $n_{\bar{F}}$ then they can be analytically continued to match in non-physical regions. We can thus take the limit $n_{F}, n_{\bar{F}} \rightarrow \infty$ at fixed ratio for arbitrary such ratio.

2. It is a composite object. In this case we expect all of its $U(1)$ charges to scale like $N$ in the dual theory, whereas they do not do so in the original theory ${ }^{1}$.

3. It does not exist. This is exactly what $W=\operatorname{Pf}(A)$ does.

Adding this superpotential, we are left with a theory that has the following $U(1)$ charges:

$\begin{array}{lll} & U(1)_{1} & U(1)_{R} \\ Q & 1 & 1+\frac{8-6 N-N^{2}}{N\left(n_{F}+n_{\bar{F}}\right)} \\ \bar{Q} & -\frac{n_{F}}{n_{\bar{F}}} & 1+\frac{8-6 N-N^{2}}{N\left(n_{F}+n_{\bar{F}}\right)} \\ A & 0 & 4 / N\end{array}$

Asymptotic freedom is lost at $6 N-n_{F}-n_{\bar{F}}-(N-2)=0$. For reasons that will be clear later, we will be interested primarily in the region $3 n_{F}-12>n_{\bar{F}}>2 n_{F}-1$ (in this region the theory is asymptotically free). Note also that in this range, at generic points along the flat directions, the gauge group is completely Higgsed.

There is no additional dynamically generated superpotential when the gauge group is completely Higgsed. In this case a dynamically generated superpotential should be understood as an instanton effect and we can expand the superpotential in instanton powers. Imposing the various symmetries then forbids any additional superpotential [35].

As in [1], one can make the following arguments in favor of the existence of a nonabelian Coulomb phase:

1. In the limit where $n_{F}$ and $n_{\bar{F}}$ very large, and $n_{F}+n_{\bar{F}}$ very close but smaller then $5 N+2$ (this region of $n_{F}$ and $n_{\bar{F}}$ is excluded from the region above, though) then the 2 loop term in the $\beta$ function can balance the 1 loop $\beta$ function, yielding a non trivial, interacting, field theory in a perturbative regime of the coupling constant [17].

\footnotetext{
1 In any case, in appendix 1 we construct the dual without assuming any $W$ and derive it
} 
2. The theory has classical flat directions in which $A=0$ and so are $n_{\bar{F}}-n_{F}$ of the antiquarks. The D-term equations are then the usual ones for $S U(N)$ with $n_{F}$ flavors. We can break $S U(N)$ to $S U(k)$ and be left with $n_{F}-N+k+(N-k)=n_{F}$ quarks $(A$ also contributes fundamentals of $S U(k)), n_{\bar{F}}-N+k=n_{F}+k-4$ antiquarks and an antisymmetric tensor. The theory is not asymptotically free when $4 k+6-2 n_{F} \leq 0$, i.e., $k_{\max } \leq \frac{n_{F}-3}{2}$. We would expect, at least, an $S U\left(k_{\max }\right)$ subgroup to be in a non-abelian Coulomb phase at the origin.

\section{The Expanded Model}

One can go and directly find other models satisfying the 't Hooft anomaly matching conditions and check if they are dual to this model $^{2}$, or one can use the following shortcut.

As described in [1], in SQCD, when $N_{f}=N_{c}+1$, mesons and baryons saturate the anomaly matching conditions, and constitute a valid description of the low energy physics. Such a point exists also for $S p\left(N_{2}\right)^{3}$ [13]. Indeed, the mesons alone saturate the anomaly, which is fortunate since the baryons decompose into an antisymmetrized product of mesons. We would therefore like to suggest that the model at hand is equivalent to the following model:

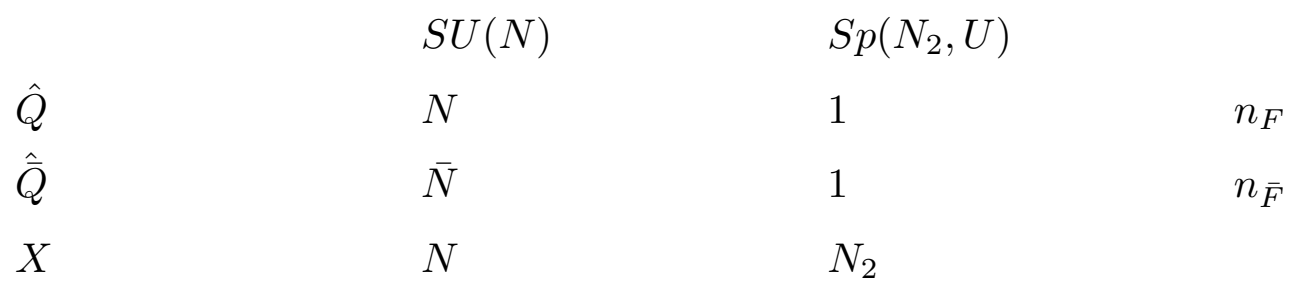

where $N=n_{\bar{F}}-n_{F}+4, N_{2}=n_{\bar{F}}-n_{F}$ and $A \sim X J X$ where $J$ is the symplectic form.

The equivalence between the two models is the following. We first solve the D-term constraints of $S p[13]$. Choose a maximal lagrangian space ${ }^{4}$ in the space spanned by the $S p$ vectors in $X$, and diagonalize that part of $X$ using $S U(N)$ and $S p\left(N_{2}\right)$ transformations.

\footnotetext{
2 Appendix 1 contains a series of arguments that determines the dual directly

3 The notation is such that $N_{2}$ is the dimension of the fundamental. The dimension of the group is then $\frac{N_{2}\left(N_{2}+1\right)}{2}$

4 A subspace of $C^{N_{2}}$ on which the symplectic form vanishes
} 
The D-term constraints then imply that we have the same diagonal values in the conjugate lagrangian subspace (up to an allowed $S U(N)$ transformation). This defines a $1-1$ correspondence between $A$, constrained by $W=\operatorname{Pf}(A)$, and $X^{2}$, with $X$ transforming as above.

This, however, is not entirely correct. This correspondence, appended with $\hat{Q}=Q$ and $\bar{Q}=\bar{Q}$, does not map $D^{S U}$ in the original theory to $D^{S U}$ in the expanded theory. This can be fixed by twisting the transformation by $A=G X^{2} G^{T}, Q=G \hat{Q}, \bar{Q}=G^{-1} \hat{\bar{Q}}$ where $G \in S L(N, C)$, depends on $Q, \bar{Q}, A$, and acts on the $S U(N)$ indices of the fields. To find $G$ we require that it intertwines the $D$-terms of the $S U(N)$ in the two theories.

More precisely: The D-term for $S U(N)$ in the original theory is

$$
-\bar{Q}^{*} \bar{Q}^{T}+Q Q^{\dagger}+2 A A^{\dagger}=c_{1} I
$$

and for the expanded theory it is

$$
-\hat{\bar{Q}}^{*} \hat{\bar{Q}}^{T}+\hat{Q} \hat{Q}^{\dagger}+X X^{\dagger}=c_{2} I
$$

and, after eliminating $Q$ and $\hat{Q}$, the equation obtained for the twist matrix is

$$
-G^{-1^{*}} \hat{\bar{Q}}^{*} \hat{\bar{Q}}^{T} G+G \hat{\bar{Q}}^{*} \hat{\bar{Q}}^{T} G^{\dagger}-G X X^{\dagger} G+2 G X^{2} G^{T} G^{*} X^{2^{\dagger}} G^{\dagger}=c_{1} I+c_{2} G G^{\dagger}
$$

There are $N^{2}$ real equations (the equation is automatically hermitian) and $N^{2}$ real unknown variables (the numbers of parameters in $G$ is $\operatorname{dim}(S L(N, C) / S U(N))$ and $\left.c_{1}\right)$. We have not been able to solve the relation, but typically we expect that there is a 1-1 map from one set of elementary quarks to another, which, by construction, does not alter the values of composite objects. Written in terms of gauge invariant objects this is a $1-1$ holomorphic map from one moduli space of vacua to the other, intertwining all constraints between gauge invariant holomorphic objects.

The physics here is that of confinement [13] and the two theories are equivalent in the I.R., only after the massive bound states in the $S p$ sector have decoupled from the theory. The gauge dynamics in $S p$ is strictly strong; the fixed point we flow to is at finite value of the $S U$ coupling constant but at infinite value of the $S p$ coupling constant. 
We suggest that a similar construction can serve as a general guideline for constructing sets of duals. For example, one could think of replacing a symmetric tensor of $S U(N)$ with an $S O(N+4)$ gauge group [14] and a new fundamental field transforming as $N \times(N+4)$ under $S U(N) \times S O(N+4)$. Indeed at $N_{c}^{\prime}=N_{f}^{\prime}+4, S O\left(N_{c}^{\prime}\right)$ has several branches of vacua. In one type of these branches there is no superpotential (and on the other there is no ground state) and the symmetric meson of this theory is the correct description in the I.R. An adjoint in $S U(N)$ may require expanding with other $S U\left(N^{\prime}\right)$ gauge groups [9 11].

\section{The Dual Theories}

At this stage one has a model which contains only (anti)fundamentals in each gauge group. We can then obtain suggestions for a dual model by first dualizing the $S U$ sector by the known $S U$ duality [1] and then dualizing the $S p$ sector by a similarly suggested duality [1] [13]; clearly this satisfies the anomaly matching conditions. It also provides the translation dictionary between bound states in the original, expanded and dual theories; given a bound state in the original theory, translate it into the expanded theory and then construct, in stages, its counterparts. When dualizing one gauge group, some of the mesons generated are charged under the second gauge group but this does not affect the translation table.

After dualizing $S U(N)$ [1] one obtains:

$\begin{array}{llll} & S U\left(N_{3}=n_{F}-4\right) & S p\left(n_{\bar{F}}-n_{F}, U\right) & \\ q_{1} & N_{3} & 1 & \bar{n}_{F} \\ q_{2} & \bar{N}_{3} & 1 & \bar{n}_{\bar{F}} \\ q_{4} & N_{3} & N_{2} & \\ q_{5} & 1 & N_{2} & n_{\bar{F}} \\ M & 1 & 1 & n_{F} \times n_{\bar{F}}\end{array}$

with a superpotential $q_{2} q_{5} q_{4}+q_{1} q_{2} M$. The notation $\bar{n}_{\bar{F}}\left(\bar{n}_{F}\right)$ denotes an anti-fundamental under the flavor group $S U\left(n_{\bar{F}}\right)\left(S U\left(n_{F}\right)\right)$.

Note that the $S p$ group is not driven to the range in which it generates a superpotential [13]. Even if $q_{2}$ has maximal rank, it can mass up $n_{F}-4$ of the $S p$ quarks at most, leaving 
$n_{\bar{F}}-n_{F}+4$ of them massless. It is remarkable, however, that by dualizing $S U$ we have already transformed $S p$ from a region with strong coupling dynamics in the flat directions to a region without. We will have more to say on this issue in the following section, but for now we notice that the choice of the range of $n_{F}, n_{\bar{F}}$ that was done before ensures that both gauge groups are asymptotically free.

One can dualize $S p$ [1] in this model and, after integrating out some degrees of freedom [4], obtain:

$\begin{array}{llll} & S U\left(n_{F}-4\right) & S p\left(N_{4}=2 n_{F}-8\right) & \\ p_{1} & N_{3} & 1 & \bar{n}_{F} \\ p_{2} & 1 & N_{4} & \bar{n}_{\bar{F}} \\ p_{3} & \left(N_{3} \times N_{3}\right)_{A} & 1 & \\ p_{4} & \bar{N}_{3} & N_{4} & \left(n_{\bar{F}} \times n_{\bar{F}}\right)_{A} \\ N & 1 & 1 & n_{F} \times n_{\bar{F}}\end{array}$

with a superpotential $N p_{2} p_{2}+M p_{1} p_{4} p_{2}+p_{3} p_{4} p_{4}$.

The last term, which is automatically generated, eliminates the meson $p_{1} p_{4}^{2} p_{1}$ from the chiral ring.

Note that the regions in which the expanded and two dual theories are asymptotically free are different (but overlapping) regions of $n_{F}$ and $n_{\bar{F}}$. One could argue that the descriptions are dual on the regions of overlap (which is the region we have been restricting ourselves to so far) but there is another possible solution to this puzzle, which we will present in the next section.

\section{Chiral Operators}

As noted above, identifications of chiral operators are almost automatic. We will demonstrate the translation for a few operators: 


$\begin{array}{lll}\text { Original theory } & \text { Expanded theory } & \text { Dual } 1 \\ Q \bar{Q} & Q \bar{Q} & M \\ \bar{Q} A \bar{Q} & \bar{Q} X^{2} \bar{Q} & q_{5}^{2} \\ \bar{Q}^{N} & \bar{Q}^{N} & q_{2}^{n_{F}-4} \\ Q^{k} A^{\frac{N-k}{2}} & Q^{k}(X J X)^{\frac{N-k}{2}} & q_{1}^{n_{F}-k}\left(q_{4} J q_{4}\right)^{\frac{k-4}{2}}\end{array}$

Translating into Dual 2 is also simple; replace any $q_{5}^{2}$ by $N$ and $q_{4}^{2}$ by $p_{3}$ (noting also that the corresponding dual mesons of the $S p$ sector, $p_{2}^{2}$ and $p_{2} p_{4}$, are redundant). Since $q_{2}$ has been integrated from the theory via its equations of motion, which are $q_{2}=p_{2} p_{4}$, the baryon $\bar{Q}^{N}$ now corresponds to $\left(p_{2} p_{4}\right)^{n_{F}-4}$.

The $U(1)$ charges of these operators are:
Original theory
$U(1)_{1}$
$U(1)_{R}$
$Q \bar{Q}$
$1-\frac{n_{F}}{n_{\bar{F}}}$
$2+2 \frac{8-6 N-N^{2}}{N\left(n_{F}+n_{\bar{F}}\right)}$
$\bar{Q} A \bar{Q}$
$-2 \frac{n_{F}}{n_{\bar{F}}}$
$\bar{Q}^{N}$
$-\frac{n_{F}}{n_{\bar{F}}} N$
$2+2 \frac{8-6 N-N^{2}}{N\left(n_{F}+n_{\bar{F}}\right)}+\frac{4}{N}$
$Q^{k} A^{\frac{N-k}{2}}$
$k$
$N+\frac{8-6 N-N^{2}}{n_{F}+n_{\bar{F}}}$
$k\left(1+\frac{8-6 N-N^{2}}{n_{F}+n_{\bar{F}}}\right)+\frac{4(N-k)}{2 N}$

It is simplest to examine these relations in the limit that $N, n_{F}, n_{\bar{F}}$ are taken to infinity at fixed ratios. In this case the charges are
Original theory
$U(1)_{1}$
$U(1)_{R}$
$Q \bar{Q}$
$1-\frac{n_{F}}{n_{\bar{F}}}$
$2-2 \frac{N}{n_{F}+n_{\bar{F}}}$
$\bar{Q} A \bar{Q}$
$-2 \frac{n_{F}}{n_{\bar{F}}}$
$\bar{Q}^{N}$
$-\frac{n_{F}}{n_{\bar{F}}} N$
$2-2 \frac{N}{n_{F}+n_{\bar{F}}}$
$Q^{k} A^{\frac{N-k}{2}}$
$k$
$N\left(1-\frac{N}{n_{F}+n_{\bar{F}}}\right)$
$k\left(1-\frac{N}{n_{F}+n_{\bar{F}}}\right)+\frac{4(N-k)}{2 N}$

and one observes that there is a continuum of $\mathrm{R}$ symmetries that are allowed as the $\mathrm{R}$ symmetry of the conformal field theory (CFT) to which the theory flows in the infrared, i.e., these choices of charges that do not violate any unitarity bound (the only restriction is $2-2 \frac{N}{n_{F}+n_{\bar{F}}}>\frac{2}{3}$ which is automatic in the region of $n_{F}, n_{\bar{F}}$ we are looking at). More 
specifically, we can define $U(1)_{R}^{\text {new }}=U(1)_{R}+c U(1)_{1}$ where $c$ is a real number, bounded from above and below by the unitarity bound [1]. To remind the reader, the unitarity bound is $D(\phi)=\frac{3}{2} R(\phi)$ where $D(\phi)$ is the scaling dimension of any gauge invariant chiral field $\phi ; D>1$ for interacting spinless gauge invariant chiral fields and $D=1$ for non interacting.

This is unlike the situation in most existing examples. For example in $S U\left(N_{c}^{\prime}\right)$ with $N_{f}^{\prime}$ flavors [1], a discrete charge conjugation makes it natural to assign equal $\mathrm{R}$ charges to quarks and antiquarks, pinpointing the $\mathrm{R}$ symmetry with which to calculate the scaling dimensions.

Note, however, that none of the allowed $\mathrm{R}$ symmetries here can be identified with the $\mathrm{R}$ symmetry in the SQCD case.

We can now also suggest another solution to the problem presented in the section before. The unitarity bound, for large $n_{F}$ and $n_{\bar{F}}$, is $n_{\bar{F}}<5 n_{F}$ (to check non leading terms we have to include the 2 nd loop term in the $\beta$ function, this contains the scaling dimensions of non gauge invariant operators, which we don't know). This is also the limit in which dual 2 looses asymptotic freedom. So one is led to conclude that the description in terms of dual 2 is valid up to that point. This is similar to what happens in other existing examples. Dual 1, however, behaves differently. The $S U$ gauge group is dual 1 apparently looses asymptotic freedon earlier, at $n_{\bar{F}}=3 n_{F}$, however, no unitarity bound is violated at this transition. One is then led to speculate that it still flows to a CFT, at a regime in which perturbation theory is meaningless, the usual calculation of asymptotic freedom is not reliable, and the theory is not free in the I.R.

\section{Flat Directions}

We will show that, in the flat directions of the expanded model and dual 1 , the submanifolds of generic points are the same. Let us for a minute neglect the D-terms of the $S p$ group. Then we just have an $S U$ theory and its dual, and the equivalence of their flat directions has been established in [1]. In our notation we have two manifolds, on one of them, $\mathcal{M}$, there is a set of coordinates $\left\{Q^{k} X^{N-k}, Q \bar{Q}, X \bar{Q}, \bar{Q}^{N}\right\}$ satisfying some set of holomorphic 
constraints and on the other one there is a set of coordinates $\left\{q_{1}^{n_{F}-k} q_{4}^{k+4}, M, q_{5}, q_{2}^{n_{F}-4}\right\}$ and, by $S U(N)$ duality, these are the same coordinates on the same manifold. The $S p$ symmetry acts on this manifold, and again by construction, $S p^{c}$ (complexified $S p$ ) acts in the same way on the two manifolds. It is now easy to see that the moduli spaces of our theories, with gauged $S p$, are isomorphic at generic points since the generic points of the moduli spaces are both the generic points of $\mathcal{M} / S p\left(N_{2}, U\right)^{c}$.

The explicit computation is straightforward. We first argue that the arguments in [1] that lead to the equivalence between $S U$ and its dual still hold. One may worry that the superpotential used in [1] is not gauge invariant under $S p$ and is not allowed in our theory. Indeed, the form presented there, althought reflecting the correct physical picture, is not $S U\left(N_{F}\right)$ invariant. This is, however, a technical point and one can write it in a flavor invariant way $^{5}$.

The next step, i.e. that of modding out by $S p^{c}$, is nothing but a global version of the super-Higgs mechanism. Pick up some $Q, \bar{Q}, X$ in the extended theory that are allowed by the $D^{S U}$. The orbits of $S p^{c}$ are given by $X \mapsto G X$ where $G \in S p^{c}$. We would like to show that in a generic orbit of $S p^{c}$ there is one orbit of $S p$ that satisfies $D^{S p}$. By looking at $S p$ gauge invariant objects (or by the Higgs mechanism), this will then give the desired result that the generic points of the moduli space of vacua are the generic points of $\mathcal{M} / S p^{c}$.

We would like to ask, therefore, whether there is a $G \in S p^{c}, G \notin S p$ such that both $X X^{\dagger}$ and $G X X^{\dagger} G^{\dagger}$ satisfy $D^{S p}$. By choosing specific gauges of $S p$, the question is whether there is such a $G$ such that

$$
\left(\begin{array}{ll}
\alpha & 0 \\
0 & \alpha
\end{array}\right)=G\left(\begin{array}{ll}
\beta & 0 \\
0 & \beta
\end{array}\right) G^{\dagger}
$$

where $\alpha$ is a real non-degenerate diagonal matrix of size $\frac{N_{3}}{2} \times \frac{N_{3}}{2}$ (non degeneracy is the requirement that the point is generic). Direct algebraic manipulations then show that $G$ in fact has to be in $S p\left(N_{2}, U\right)$.

The same argument holds for dual 1, concluding the proof.

5 We keep the massive quarks in order to make the flavor symmetry manifest and then integrate them out. 


\section{Adding mass perturbations}

We add to the original theory a perturbation $m Q_{1} \bar{Q}_{1}$ and flow to a theory with one $n_{F}^{n e w}=n_{F}-1, n_{\bar{F}}^{n e w}=n_{\bar{F}}-1$. In the dual theories we add a perturbation $m M_{1,1}$. We would like to show that the dual theory flows to the dual theory with $n_{F}^{n e w}, n_{\bar{F}}^{n e w}$.

For the first dual the computation is identical to one studied by Seiberg in [1] for $S U(N)$ theories.

In the second dual the computation is the following. We add a perturbation $M_{1,1}$, choose the most symmetric combination and expand about it. The configuration we choose is

$$
p_{1,1}=\left(\begin{array}{c}
1 \\
0 \\
\cdot \\
.
\end{array}\right), p_{2,1}=\left(\begin{array}{c}
1 \\
\overrightarrow{0} \\
0 \\
\overrightarrow{0}
\end{array}\right), p_{4,\left(N_{4} \times N_{3}\right)}=\left(\begin{array}{cccc}
0 & 0 & \ldots & 0 \\
\overrightarrow{0} & 0 & \ldots & 0 \\
1 & 0 & \ldots & 0 \\
\overrightarrow{0} & 0 & \ldots & 0
\end{array}\right)
$$

The complexification of $\operatorname{sp}(2 n, U)$ is

$$
\left(\begin{array}{cc}
A & B \\
C & -A^{T}
\end{array}\right)
$$

$B^{T}=B, C^{T}=C$

We wish to discuss its action on pair of vectors

$$
\left(\left(\begin{array}{c}
1 \\
\overrightarrow{0} \\
0 \\
\overrightarrow{0}
\end{array}\right),\left(\begin{array}{c}
0 \\
\overrightarrow{0} \\
1 \\
\overrightarrow{0}
\end{array}\right)\right)
$$

where we have divided $C^{N_{4}}$ into 2 lagrangian subspaces of dimension $N_{4} / 2$ (two upper components vs. two lower components) and in each subspace we have separated the first coordinate from the rest.

The relevant span is

$$
\begin{gathered}
\left(\begin{array}{cc}
A & B \\
C & -A^{T}
\end{array}\right)\left(\begin{array}{l}
1 \\
\overrightarrow{0} \\
0 \\
\overrightarrow{0}
\end{array}\right)=\left(\begin{array}{c}
a \\
c
\end{array}\right) \\
\left(\begin{array}{cc}
A & B \\
C & -A^{T}
\end{array}\right)\left(\begin{array}{l}
0 \\
\overrightarrow{0} \\
1 \\
\overrightarrow{0}
\end{array}\right)=\left(\begin{array}{c}
b \\
-\hat{a}
\end{array}\right)
\end{gathered}
$$


where $a, \hat{a}, c, c$ are arbitrary vectors in $C^{\frac{N_{4}}{2}}$ with the single constaint $a_{1}=\hat{a}_{1}$.

One sees that the only direction that does not receive mass in the Higgs mechanism in $S p$ is the direction

$$
\lambda\left(\left(\begin{array}{l}
1 \\
\overrightarrow{0} \\
0 \\
\overrightarrow{0}
\end{array}\right),\left(\begin{array}{l}
0 \\
\overrightarrow{0} \\
1 \\
\overrightarrow{0}
\end{array}\right)\right), \lambda \in C
$$

This direction is massed up together with $M_{11}$.

After taking into account the directions that are Higgsed by $S U$ breaking we are left with gauge groups that are broken to $S U\left(N_{4}\right) \rightarrow S U\left(N_{4}-1\right), S p\left(N_{3}\right) \rightarrow S p\left(N_{3}-2\right)$.

$$
\begin{aligned}
& p_{1,\left(N_{4} \times n_{F}-1\right)}=\left(\begin{array}{ccc}
\leftarrow & v & \rightarrow \\
& & \\
& p_{1}^{\prime} &
\end{array}\right), p_{2,\left(N_{3} \times n_{\bar{F}}-1\right)}=\left(\begin{array}{ccc}
\leftarrow & w & \rightarrow \\
& p_{2}^{\prime} & \\
\leftarrow & t & \rightarrow \\
& p_{2}^{\prime}
\end{array}\right) \\
& p_{4,\left(N_{4} \times N_{3}\right)}=\left(\begin{array}{ccccc}
h & \leftarrow & s & \rightarrow \\
i & & p_{4}^{\prime} & \\
g & & & \\
1 & \leftarrow & \text { higgs } & \rightarrow \\
g & & p_{4}^{\prime} & \\
s & & &
\end{array}\right), p_{3}=\left(\begin{array}{cccc}
0 & \leftarrow & z & \rightarrow \\
\uparrow & & & \\
-z & & p_{3}^{\prime} & \\
\downarrow & & &
\end{array}\right) \\
& M_{\left(n_{F} \times n_{\bar{F}}\right)}=\left(\begin{array}{cccc}
M_{1,1} & \leftarrow & c & \rightarrow \\
\uparrow & & & \\
e & & M^{\prime} & \\
\downarrow & & &
\end{array}\right), N_{\left(n_{\bar{F}} \times n_{\bar{F}}\right)}=\left(\begin{array}{cccc}
0 & \leftarrow & b & \rightarrow \\
\uparrow & & & \\
-b & & N^{\prime} & \\
\downarrow & & &
\end{array}\right)
\end{aligned}
$$

Examining the superpotential, $N p_{2} p_{2}$ spawns the mass term $t b, M p_{1} p_{2} p_{4}$ spawns $e v$ and $v s$ (and the mass term for $M_{1,1}$ ) and $p_{3} p_{4} p_{4}$ spawns $s z$.

\section{Discussion}

We have suggested a dual to an $S U(2 k)$ Susy gauge theory containing an antisymmetric tensor, quarks and antiquarks. This model contains some new features:

1. The dual contains 2 gauge groups and does not look similar to the original model.

2. There are no discrete symmetries that fix a specific $R$ symmetry as the one determining the scaling dimenstions. 
3. The duality transformation can be understood in terms of other, simpler, duality transformations. One may therefore expect to have a set of "primitive dualities" that can be composed together after expanding the models and rewriting complicated representations as confined objects built out of simpler elementary fields. This procedure of expansion of a gauge theory with complicated representations into one with more gauge groups, but all matter fields in the fundamental, may be of more general utility.

We hope this example will be useful in gaining a deeper understanding of duality.

\section{Acknowledgments}

It is a pleasure to thank T. Banks, M. Dine, K. Intriligator and P. Pouliot for illuminating discussions and comments, and to thank SLAC and the Weizmann Institute Of Science for their hospitality while completing this work.

\section{Appendix 1}

One can construct the 2nd dual directly. As claimed above we will not take the baryon $\operatorname{Pf}(A) Q A^{-1} Q$ to be an elementary excitation in the dual theory so we are left with $Q \bar{Q}_{n_{F} \times n_{\bar{F}}}$ and $\bar{Q} A \bar{Q}_{\left(n_{\bar{F}} \times n_{\bar{F}}\right)_{A}}$ as natural candidates.

Let us make the following assumptions:

1. All the dual quarks transform as anti-fundamentals under the flavor groups. We then know that the dual quarks are in different gauge groups and the size of the groups.

2. The superpotential is of the form $W=\sum M_{i}^{D} M_{i}+W_{2}$ where $M^{D}(M)$ are mesons in the dual (original) theory and $W_{2}$ is independent of $M$ and of the dual quarks. We are not assuming a potential $\operatorname{Pf}(A)$ in the original theory, and we will derive it.

$M_{i}^{D}$ are all the mesons in the dual theory (which are not zero by other equations of motion). These must be in 1-1 correspondence to the mesons, $M_{i}$, taken from the original theory (which are all the mesons in the original theory not set to zero by the equations of motion there). We cannot have more $M_{i}^{D}$ since then we would have mesons in the dual theory that are not redundant but are not in the electric theory, and we can not have more $M_{i}$ since then we will have a non-interacting (electric) meson the dual theory whereas we can not identify such a non-interacting meson in the original theory. We are 
assuming here that a field cannot be made interacting only through the Kähler potential (and we will also assume that the Kähler potential does not break symmetries apparent in the superpotential).

The assumption on $W_{2}$ is a simplifying assumption. We can actually make do with a weaker assumption that the equations of motion originating from $W_{2}$ do not set to zero any dual mesons, and that therefore the only way of eliminating them from the chiral ring is by coupling them to mesons from the original theory. This is the case in existing examples of duality.

By now we know the sizes of the groups and also have information on their type. Since we don't have any electric meson in $\left(n_{F} \times n_{F}\right)_{A}$ we must forbid dual quarks, $q_{D}$, transforming as $\bar{n}_{F}$ to bind into a gauge invariant mesons $q_{D} q_{D}$, so $q_{D}$ must transform under an $S U$ group gauge group. Since there is no way to cancel the anomaly in the gauge group containing the dual quarks transforming as $\bar{n}_{\bar{F}}$ (without adding more gauge groups) it must be an $S p$ group. (We argue that there is also one dual quark that transforms under $S U\left(n_{\bar{F}}\right)$. Suppose there are two such dual quarks. If there is no dual meson built from these then we have an $S U\left(n_{\bar{F}}\right) \times S U\left(n_{\bar{F}}\right)$ symmetry. If we have two such quark then the dual meson built from them has no particular symmetry properties. It must couple to an original $n_{\bar{F}} \times n_{\bar{F}}$ meson that contains both the symmetric and the antisymmetric part and again we have an $S U\left(n_{\bar{F}}\right) \times S U\left(n_{\bar{F}}\right)$ symmetry in the superpotential.)

So far we have the following fields in the suggested dual

$\begin{array}{llll} & S U\left(N_{3}=n_{F}-4\right) & S p\left(N_{4}=2 n_{F}-8\right) & \\ p_{1} & N_{3} & 1 & \bar{n}_{F} \\ p_{2} & 1 & N_{2} & n_{F} \\ M & 1 & 1 & n_{F} \times n_{\bar{F}} \\ N & 1 & 1 & \left(n_{\bar{F}} \times n_{\bar{F}}\right)_{A}\end{array}$

Denote $l_{1}=$ number of fields $N_{3} \times N_{3}$ - number of fields $\bar{N}_{3} \times \bar{N}_{3}, l_{2}=k^{+}-k^{-}$, where in $k^{ \pm}$counts the number of fields that transform under $S U\left(N_{3}\right) \times S p\left(N_{4}\right)$ such that a $+(-)$ is an (anti)fundamental under $S U\left(N_{3}\right)$. We will assume that $l_{1}$ and $l_{2}$ are fixed as we vary $n_{F}$ and $n_{\bar{F}}$. In principal the change can be of the form $\left\lfloor f\left(n_{F}, n_{\bar{F}}\right)\right\rfloor$ but then the 
equations for the anomaly matching will not be algebraic and it is not clear how to deal with them.

Define the following $\mathrm{U}(1)$ symmetry: In $S U\left(N_{3}\right)$, to every field, except $p_{1}$, give charge +1 if it has an $N_{3}$ index and -1 if it has an $\bar{N}_{3}$ index. Cancel the anomalies with $p_{1}$ and $p_{2}$. $N$ and $M$ have charges $-2 p_{2}$ and $1-p_{1}-p_{2}$ (charges are denoted with $p_{1}$ etc.).

The $U(1)$ above is some $\alpha U(1)_{1}+\beta U(1)_{2}$ (where the charges under $U(1)_{2}$ in the electric theory are $\left.A_{(1)}, Q, \bar{Q}_{\left(\frac{2-N}{\left(n_{F}+n_{F}\right.}\right)}\right)$ in the electric theory. Then the charges of mesons imply

$$
\begin{gathered}
-\frac{2 n_{F}}{n_{\bar{F}}} \alpha+\left(1-2 \frac{N-2}{n_{F}+n_{\bar{F}}}\right) \beta=-2 p_{2} \\
\left(1-\frac{n_{F}}{n_{\bar{F}}}\right) \alpha-2 \frac{N-2}{n_{F}+n_{\bar{F}}} \beta=1-p_{1}-p_{2}
\end{gathered}
$$

from which one obtains

$$
\beta=-\frac{2}{N}\left(n_{F}+l_{2} N_{3}+2 l_{1} N_{3}+l_{2} N_{4}\right)
$$

in the limit that $n_{F}, n_{\bar{F}} \rightarrow \infty$ at fixed ratio, which we will assume from now on.

Already we can see that we will run into problems with this $\beta$. Currently it is of the form $\frac{a n_{F}+b n_{\bar{F}}}{N}$. We have to cancel the $N$ in the denominator, otherwise there will be a $\frac{1}{N}$ pole in $\sum U(1)^{3}$ electric, but there is no such pole in the magnetic theory (because these are algebraic equations we can analytically continue them to a non-physical regime, in this case $N=0$ ). After eliminating the pole in $N$ (which fixes $b=-a$ ) we are left with a $\frac{1}{n_{F}+n_{\bar{F}}}$ pole in $\sum U(1)^{3}$ electric that does not exist in the magnetic. Thus $\beta=0$. So we have identified $U(1)_{1}$.

Using the above equations with $\beta=0$, and the equations for $\sum U(1)$ as equations for $\alpha, l_{1}, l_{2}$ (where $\alpha$ may depend on $n_{F}$ and $n_{\bar{F}}$ but $l_{1}$ and $l_{2}$ may not) one obtains $l_{1}=1, l_{2}=-1$. Since we don't want any $\bar{n}_{F} \times \bar{n}_{F}$ meson, we have no fields of the form $\bar{N}_{3} \times \bar{N}_{3}$, fixing $p_{3}$ in the 2 nd dual. We have no compelling argument why the solution to the constraint $l_{2}=-1$ is just with one $p_{4}$, it is certainly the simplest solution. We have tried other ways of solving the constraint (one has the freedom to add adjoints also) and typically one has magnetic mesons that are not in the electric theory or too many $U(1)$ in the magnetic theory (long before checking flat directions). After having the solution one 
can argue the following. Since we have matched the chiral ring with just one $p_{4}$, all gauge invariant objects we build with whatever additional field have to be zero by the equations of motion. Very likely this means that we can consistently set all the additional fields to zero and forget about them. 


\section{References}

[1] N. Seiberg, hep-th/9411149, RU-94-82, IASSNS-HEP-94/98

[2] I. Affleck, M. Dine and N. Seiberg, Nucl. Phys. B241 (1984) 493; Nucl. Phys. B256 (1985) 557

[3] N. Seiberg, hep-ph/9309335, Phys. Lett. 318B (1993) 469

[4] N. Seiberg, hep-th/9402044, Phys. Rev. D49 (1994) 6857

[5] K. Intriligator, R.G. Leigh and N. Seiberg, hep-th/9403198, Phys. Rev. D50 (1994) 1092; K. Intriligator, hep-th/9407106, Phys. Lett. 336B (1994) 409

[6] N. Seiberg and E. Witten, hep-th/9407087, Nucl. Phys. B426 (1994) 19

[7] N. Seiberg and E. Witten, hep-th/9408099, Nucl. Phys. B431 (1994) 484

[8] K. Intriligator and N. Seiberg, hep-th/9408155, Nucl. Phys. B431 (1994) 551

[9] D. Kutasov, hep-th/9503086, EFI-95-11

[10] O. Aharony, J. Sonnenschein and S. Yankielowicz, hep-th/9502013, TAUP-2232-95

[11] D. Kutasov and A. Schwimmer, EFI-95-11, WIS/4/95, hep-th/9505004

[12] R. Leigh and M. Strassler, RU-95-2, hep-th/9503121

[13] K. Intriligator and P. Pouliot, RU-95-23, hep-th/9505006

[14] K. Intriligator and N. Seiberg, RU-95-3, IASSNS-HEP-95/5, hep-th/9503179

[15] N. Seiberg, The Power of Holomorphy - Exact Results in 4D SUSY Field Theories. To appear in the Proc. of PASCOS 94. hep-th/9408013, RU-94-64, IASSNS-HEP-94/57

[16] H. Osborn, Phys. Lett. 83B (1979) 321;A. Sen, hep-th/9402032, Phys. Lett. 329B (1994) 217; C. Vafa and E. Witten, hep-th/9408074, Nucl. Phys. B432 (1994) 3

[17] T. Banks and A. Zaks, Nucl. Phys. B196 (1982) 189

[18] V.A. Novikov, M.A. Shifman, A. I. Vainshtein and V. I. Zakharov, Nucl. Phys. B223 (1983) 445; Nucl. Phys. B260 (1985) 157; Nucl. Phys. B229 (1983) 381

[19] D. Amati, K. Konishi, Y. Meurice, G.C. Rossi and G. Veneziano, Phys. Rep. 162 (1988) 169 and references therein

[20] M.A. Shifman and A. I. Vainshtein, Nucl. Phys. B277 (1986) 456; Nucl. Phys. B359 (1991) 571

[21] C. Montonen and D. Olive, Phys. Lett. 72B (1977) 117 